OPEN

SUBJECT AREAS:

ELECTRONIC PROPERTIES

AND DEVICES

ELECTRONIC DEVICES

Received

22 August 2013

Accepted

27 December 2013

Published

20 January 2014

Correspondence and requests for materials should be addressed to H.K.K. (hkk@pitt.edu)

\footnotetext{
* These authors contributed equally to this work.
}

$\uparrow$ Current address: Department of

Electrical Engineering, Kasetsart University, 50 Phaholyothin Rd, Bangkok, Thailand 10900.

\section{Space charge neutralization by electron-transparent suspended graphene}

\author{
Siwapon Srisonphan*†, Myungji Kim* \& Hong Koo Kim
}

Department of Electrical and Computer Engineering and Petersen Institute of NanoScience and Engineering, 1140 Benedum, University of Pittsburgh, Pittsburgh, Pennsylvania 15261, United States of America.

Graphene possesses many fascinating properties originating from the manifold potential for interactions at electronic, atomic, or molecular levels. Here we report measurement of electron transparency and hole charge induction response of a suspended graphene anode on top of a void channel formed in a $\mathrm{SiO}_{2} / \mathrm{Si}$ substrate. A two-dimensional (2D) electron gas induced at the oxide interface emits into air and makes a ballistic transport toward the suspended graphene. A small fraction $(>\sim 0.1 \%)$ of impinging electrons are captured at the edge of 2D hole system in graphene, demonstrating good transparency to very low energy $(<3 \mathrm{eV})$ electrons. The hole charges induced in the suspended graphene anode have the effect of neutralizing the electron space charge in the void channel. This charge compensation dramatically enhances 2D electron gas emission at cathode to the level far surpassing the Child-Langmuir's space-charge-limited emission.

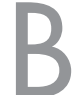

eing a $2 \mathrm{D}$ atomic crystal $^{1,2}$, graphene is transmissive to impinging electrons while being impermeable to atoms and molecules ${ }^{3}$. Harboring a $2 \mathrm{D}$ electron system $^{4-7}$, graphene can be highly conductive in in-plane transport and is expected to be interactive with out-of-plane incident electrons as well. At electron energy $<\sim 10 \mathrm{eV}$ the de Broglie wavelength becomes greater than the lattice atomic spacing and crystalline diffraction is less likely to occur. Below 5-10 eV, the dominant scattering mechanism is expected to involve inelastic interactions such as electron excitations or electron-phonon interactions ${ }^{8-10}$. The damage threshold of graphene is known to be $>15 \mathrm{eV}$, corresponding to incident electron energy $>80 \mathrm{keV}^{11,12}$. Considering the relatively large threshold, electrons of very low energy $(<3 \mathrm{eV})$ are expected to induce no damage to graphene.

A graphene electrode suspended on a nanoscale void channel provides an interesting configuration to investigate the interplay of in- and out-of-plane interactions of 2D electron systems mediated by electron transport in vacuum. In this study we characterize the emission, capture and transmission interactions with very low energy electrons and explore the potential to use graphene as an electron-transparent grid in low-voltage nanoscale vacuum electronic devices ${ }^{13-18}$.

In generating a constant flux of very low energy electrons we exploit the phenomenon that a two-dimensional electron gas (2DEG) induced at the $\mathrm{SiO}_{2} / \mathrm{Si}$ interface of a metal-oxide-semiconductor (MOS) structure can easily emit into air (void channel) at low voltage $(\sim 1 \mathrm{~V})^{18}$. This low-voltage emission, enabled by Coulombic repulsion of electrons in 2DEG, has the effect of negative electron affinity, and demonstrates high current density emission $\left(\sim 10^{5} \mathrm{~A} / \mathrm{cm}^{2}\right)$. The emitted electrons ballistically travel in the nano-void channel. The channel length (i.e., the thickness of oxide layer) is designed to be smaller than the mean free path of electrons in air ( $\sim 60 \mathrm{~nm})$. Therefore, emitted electrons should travel scattering-free in the ambient (air) channel, as if in a vacuum. The transit time is estimated to be $10-100$ fs for $10-20 \mathrm{~nm}$-thick $\mathrm{SiO}_{2}$ at 1-10 V bias.

\section{Results}

A graphene membrane was placed on top of a void channel (500 nm $\times 500 \mathrm{~nm}$ cross-section; $1 \mu \mathrm{m}$ depth) that was focused-ion-beam (FIB) etched into a $\mathrm{SiO}_{2}(23 \mathrm{~nm}$ thickness)/n- $\mathrm{Si}(5 \Omega$-cm resistivity) substrate (Fig. 1a, b; also see Supplementary Fig. 1). A graphene/oxide(or air)/Si (GOS) structure, instead of MOS, was formed by introducing a monolayer graphene as a counter-electrode to the $2 \mathrm{DEG}$ layer at the $\mathrm{SiO}_{2} / \mathrm{Si}$ interface. Here in the two-terminal mode of operation, the graphene serves as an anode while the $\mathrm{n}$-Si substrate serves as a cathode. Under forward bias (i.e., graphene electrode positively biased with respect to $\mathrm{n}$-Si substrate) a quasi-2DEG (accumulation) develops in the Si side while a 2D hole system (2DHS) forms in the graphene side (Fig. 1c). 

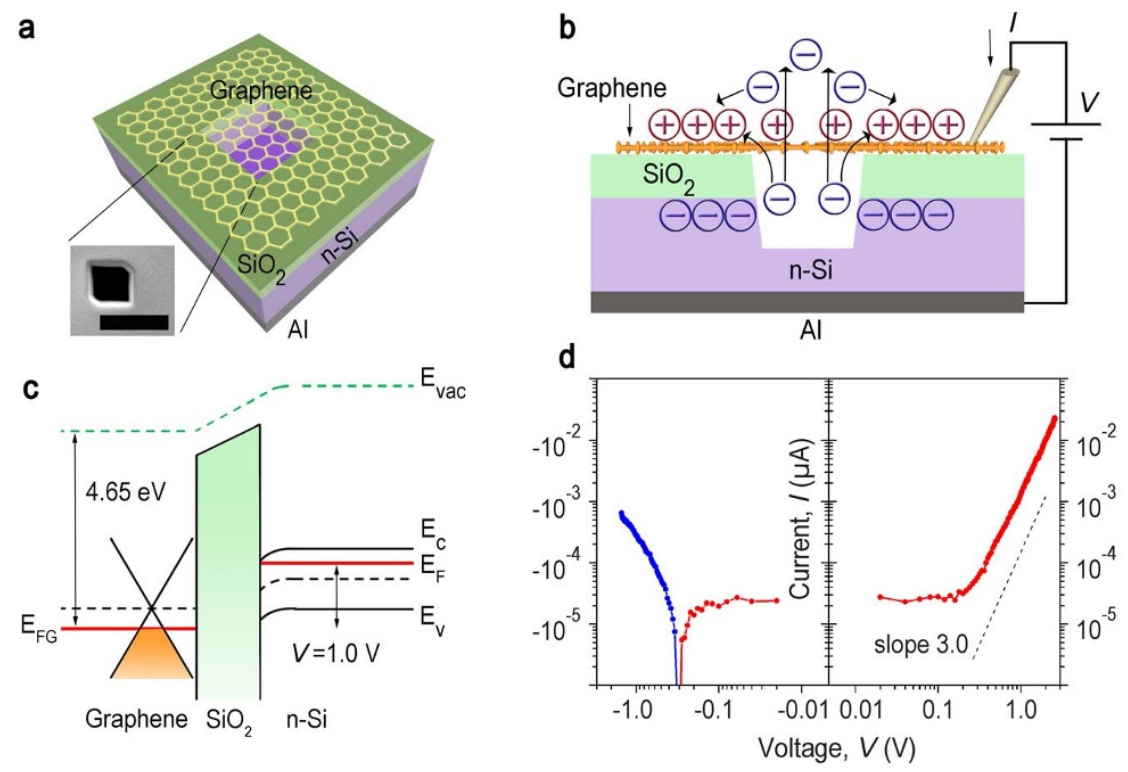

Figure 1 Transport of very low energy $(<3 \mathrm{eV})$ electrons in a void channel covered with a suspended graphene. (a), $\mathrm{Schematic} \mathrm{of} \mathrm{a} \mathrm{graphene/SiO} / 2 / \mathrm{Si}$ structure with a void channel and plan-view SEM image (inset) of a square well $\left(500 \times 500 \mathrm{~nm}^{2}\right)$ etched into $1 \mu \mathrm{m} \mathrm{depth}$ by FIB. SiO $\mathrm{F}_{2}$ thickness, $23 \mathrm{~nm}$. Scale bar, $1 \mu \mathrm{m}$. (b), Schematic of electron emission from the $2 \mathrm{DEG}$ at $\mathrm{SiO}_{2} / \mathrm{n}-\mathrm{Si}$ interface and capture/transmission at the graphene anode. (c), Schematic energy band diagram of a graphene $/ \mathrm{SiO}_{2} / \mathrm{n}$-Si structure at $1 \mathrm{~V}$ bias. (d), Measured $I-V$ characteristic of a void channel (500-nm square well) covered with a monolayer graphene. The forward $I-V$ shows $V^{3}$ dependence (red, $V>0.3 \mathrm{~V}$ ) indicating neutralization of electron space charge by holes induced in suspended graphene.

Due to Coulombic repulsion of electrons around the aperture edge, the 2DEG in Si emits into air and travels up toward the edge of 2DHS at graphene $/ \mathrm{SiO}_{2}$ interface (Fig. 1b). Some of the incident electrons are captured at the graphene, while others transmit through, forming a space charge region outside the graphene. It should be noted that in this work no external collector is employed other than the graphene anode ${ }^{19-21}$. Therefore the electrons transmitted through graphene are Coulombically attracted to and collected by the positively biased graphene anode on $\mathrm{SiO}_{2}$ surface, satisfying the charge neutrality of the overall configuration. The closed-circuit nature (i.e., charge conservation) of this two-terminal operation is confirmed by performing measurements of anode current with the system ground connected to the bottom (cathode) or top (anode) electrode, which demonstrate the same amount of channel current for given bias voltage.

Fig. 1d shows a measurement result of the channel-current-versus-voltage $(I-V)$ characteristic. The forward $I-V$ characteristic reveals the $V^{3}$ dependence for $V>0.3 \mathrm{~V}$. Note that the flat band voltage of this GOS structure is $0.25 \mathrm{~V}$, and an electron accumulation layer begins to develop at around this voltage. At $1 \mathrm{~V}$ bias, the channel current is measured to be $1.3 \mathrm{nA}$. The $V^{3}$ regime is called the double injection or injected plasma regime ${ }^{22}$. This corresponds to another type of space-charge-limited emission, differing from the Child-Langmuir's $V^{1.5}$ dependence or the Mott-Gurney's $V^{2.0}$ dependence ${ }^{13,14,23,24}$ : the $V^{3}$ regime involves bipolar space charges (electrons and holes) injected into a void channel, whereas the latter ones are mostly governed by unipolar space charges (electrons).

Presence of a free-standing graphene layer in a void channel, therefore the availability of holes in the aperture region is expected to affect the space charge field in the channel. In response to electron injection from cathode, for example, the graphene anode brings positive space charges into the void channel by inducing hole charges in the free-standing cover. This has the effect of neutralizing the electron space charges in transit in the channel region ${ }^{25,26}$. With a reduced space charge field on cathode surface, electron emission becomes easier, resulting in higher channel current with stronger voltage dependence (i.e., $V^{3}$ instead of $V^{1.5}$ or $V^{2.0}$ ). In this bipolar space-charge regime $\left(V^{3}\right)$ the cathode emission is governed by the availability of hole space charges on graphene $\left(Q_{h}\right)$. Since the carrier density in graphene has a quadratic dependence on Fermi energy $\left(n \sim E_{F}^{2}\right)^{27,28}$, the amount of electrons being injected into a channel $\left(Q_{e}\right)$ is expected to show the same voltage dependence $\left(Q_{e} \sim Q_{h} \sim\right.$ $V^{2}$ ). With enhanced injection of charge carriers in the channel region, electron transport is expected to show an average velocity that is proportional to electric field, therefore, bias voltage: $v_{a v} \sim \mu \mathcal{E} \sim$ $\mu V / L$. Here $\mu$ is the electron mobility and $L$ is the channel length. The average transit time of electron in the channel is then determined as $\tau_{a v}=L / v_{a v}$, and the channel current can be expressed as $I=Q_{e} / \tau_{a v}$ $=Q_{e} v_{a v} / L \sim V^{3}$.

Besides altering the behavior of space-charge-limited emission under forward bias, a free-standing graphene appears to affect the reverse characteristic as well (Fig. 1d). At around $-0.3 \mathrm{~V}$ the current level drops to zero, switching the polarity from reverse (blue) to forward (red). Note that bias voltage was swept in the positive direction from $-1.5 \mathrm{~V}$ to $+1.5 \mathrm{~V}$. The early reversal of current flow suggests a discharge of graphene anode around this bias. As the Fermi level is reduced toward the Dirac point, the carrier density of graphene monotonically decreases ${ }^{29}$. Electrons then evacuate from the graphene at reduced bias, and this exiting (discharging) electron flow has an effect of compensating the reverse leakage (charging) current (Supplementary Fig. 2). At some bias point the two current components cancel each other, causing a zero-current crossing (i.e., a dip in $I-V)$.

In order to estimate the electron capture efficiency at the edge of graphene anode, the total electron emission from cathode needs to be measured. To measure cathode emission, the graphene anode was covered by placing a Ga droplet in the aperture area (Fig. 2a). Note that the Ga droplet size is designed to be much larger than the channel diameter (i.e., $500 \mu \mathrm{m}$ versus $500 \mathrm{~nm}$ ) so that incident electrons are fully blocked by the Ga-covered graphene anode. The forward $I-V$ characteristic clearly reveals the $V^{3}$ dependence for $V>$ $0.1 \mathrm{~V}$. By placing Ga on top, graphene's work function is expected to decrease slightly by $\sim 0.2 \mathrm{eV}^{30}$. This will then reduce the flat band voltage to $\sim 0.12 \mathrm{~V}$, as seen in the earlier on-set of steeply rising channel current (Fig. 2b, red). The $V^{3}$ regime of the Ga-covered 
a

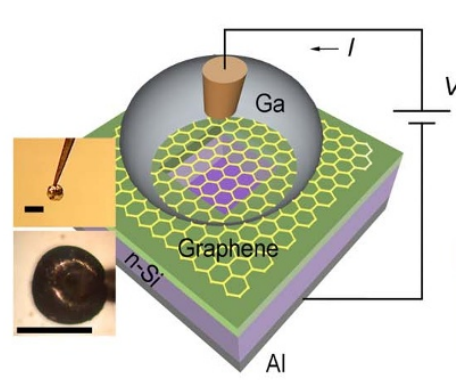

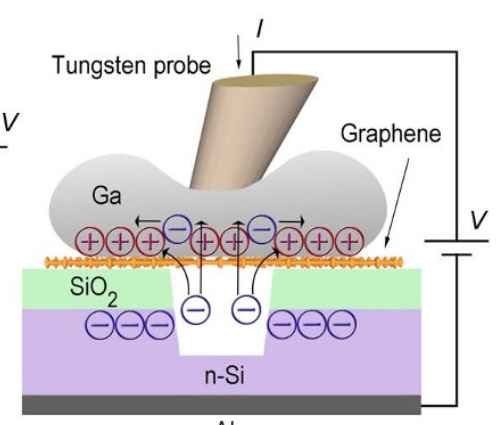

Al

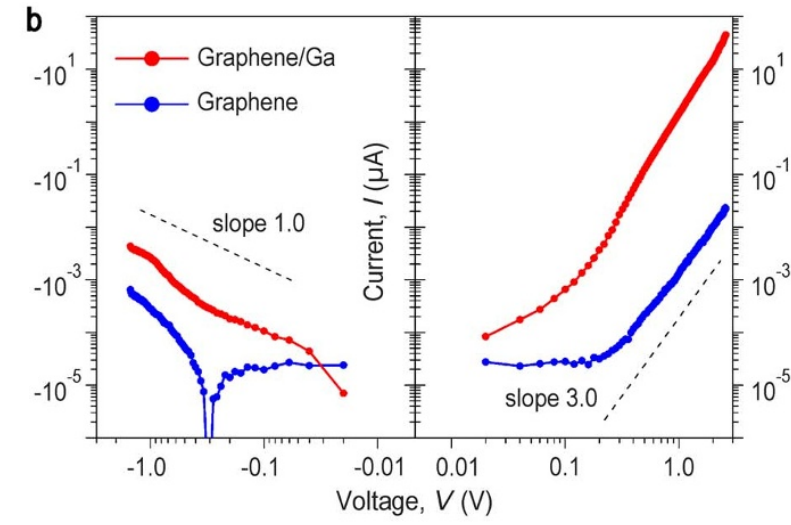

Figure 2 Electron capture efficiency of a suspended graphene anode. (a), Schematic of electron emission measurement. A Ga droplet is placed on top of the graphene anode covering the entire aperture area (inset: optical micrograph of a Ga droplet attached to a tungsten probe). Scale bars, $500 \mu \mathrm{m}$. (b), Measured $I-V$ characteristics: with a Ga cover on graphene (red) and without Ga (i.e., graphene only) (blue). The channel current increased by 1100 times (from $1.3 \mathrm{nA}$ to $1.4 \mu \mathrm{A}$ at $1 \mathrm{~V}$ bias) after placing a Ga cover, implying that the electron capture efficiency at a suspended graphene anode is estimated to be $>\sim 0.1 \%$.

graphene sample shifted up almost parallel to that of the sample without Ga. This indicates that the graphene layer underneath $\mathrm{Ga}$ still plays the same role of neutralizing electron space charge in the channel as in the case of the graphene-only sample via inducing holes in the suspended area. At $1 \mathrm{~V}$ bias, the channel current with graphene/Ga is now measured to be $1.4 \mu \mathrm{A}, 1.1 \times 10^{3}$ times increase from the current without $\mathrm{Ga}(1.3 \mathrm{nA})$. When assuming all incident electrons are blocked and captured by the graphene/Ga anode, the total electron emission from cathode equals the measured anode current $(1.4 \mu \mathrm{A})$. (Here we note that the reflectivity of very low energy electrons $(\sim 1 \mathrm{eV})$ at bulk metal surface is known to be $\sim 10 \%{ }^{10,31}$. When the reflection effect at Ga surface is taken into account, the total electron emission from cathode is expected to be $\sim 10 \%$ greater than the measured anode current.) If it is further assumed that the emission current of the graphene/Ga sample remains the same as that of the graphene-only sample, the electron capture efficiency of suspended graphene anode is estimated to be $\sim 0.1 \%$ at $1 \mathrm{~V}$ bias. By placing a Ga cover on graphene, however, the space charges that might be present in air outside the graphene are expected to be eliminated, and this may further reduce the space charge field in the channel region. Taking this possible effect into account, we note that the actual cathode emission without Ga might be less than the measured anode current with Ga. Based on this reasoning the estimated capture efficiency $(\sim 0.1 \%$ at $1 \mathrm{~V})$ should be considered as a lower limit.

The $1.4 \mu \mathrm{A}$ channel current of the graphene/Ga sample at $+1 \mathrm{~V}$ corresponds to an injection rate of $\sim 10^{13}$ electrons/s at cathode and the same rate of electron capture at Ga-covered graphene anode. The electron transit time in a nano-void channel (channel length, $23 \mathrm{~nm}$ ) is estimated to be $\sim 100 \mathrm{fs}$ at $1 \mathrm{~V}$ bias $^{22}$. This implies that on the average one electron is in transit inside the void channel. In other words, an average amount of electron space charge is to be of single electron level. A similar amount of hole charges are expected to be induced on the suspended graphene area $(500 \mathrm{~nm} \times 500 \mathrm{~nm})$. The resulting space-charge density in graphene is then estimated to be maximum $\sim 4$ holes $/ \mu \mathrm{m}^{2}$ or $\sim 4 \times 10^{8}$ holes $/ \mathrm{cm}^{2}$. The induction of holes at this level of density is expected to shift the graphene's Fermi level by no more than $0.1 \mathrm{eV}$ at $1 \mathrm{~V}$ bias $^{32-34}$. Overall the result demonstrates the graphene's enabling nature of enhancing cathode emission by inducing hole space charge at single electron level, thereby overcoming the Child-Langmuir's space charge limit ${ }^{13,14}$.

Note that the zero-current crossing point in reverse bias (i.e., the dip at $-0.3 \mathrm{~V}$ in the graphene-only sample) now shifted close to $0 \mathrm{~V}$ $(\sim-0.01 \mathrm{~V})$ with the graphene/Ga sample (Fig. 2b, red). This is explained by that the Ga-covered-graphene has a reduced work func- tion (Fermi level), shifting the discharging of graphene to occur at lower bias. For a given bias voltage, the Fermi level shift at anode might have affected the band bending in the Si side, altering the density of $2 \mathrm{DEG}$ at $\mathrm{SiO}_{2} / \mathrm{Si}$, therefore cathode emission. In order to further investigate these possible effects of anode work function change (i.e., graphene Fermi level shift) on emission and capture at 2DES edges, an additional sample structure was prepared and characterized.

Without involving graphene a Ga droplet was directly placed on top of a void-channel-etched $\mathrm{SiO}_{2} / \mathrm{n}$-Si substrate, and the resulting $I$ $V$ characteristic was compared with that of the sample with graphene/Ga (Fig. 3a, b). Again the Ga droplet size was designed to be significantly greater than the channel diameter $(500 \mu \mathrm{m}$ versus $500 \mathrm{~nm}$ ) so that incident electrons are fully captured. The forward $I-V$ characteristic reveals the $V^{2}$ dependence for $V>0.1 \mathrm{~V}$ (Fig. $3 \mathrm{c}$, red). Without space charge neutralization in the void channel (i.e., without graphene), the voltage dependence of electron injection $\left(Q_{e}\right.$ versus $V$ ) follows the capacitor relationship and is expressed as $Q_{e} \sim$ $V$. With enhanced injection of electrons into the confined space, the electron transport can be expressed in terms of average velocity $v_{a v} \sim \mu \mathcal{E} \sim \mu V / L$. The channel current is then determined as $I=$ $Q_{e} / \tau_{a v}=Q_{e} v_{a v} / L \sim V^{2}$. At low bias $(V<0.8 \mathrm{~V})$ the sample with Ga-only (red) shows larger current than the sample with graphene/ $\mathrm{Ga}$ (blue). This is explained by the fact that the work function of $\mathrm{Ga}$ $(4.3 \mathrm{eV})$ is smaller than that of the graphene under $\mathrm{Ga}$ (estimated to be $4.43 \mathrm{eV})^{30}$, and therefore accumulation electrons build up more readily at low voltage for the Ga-only sample case. At $0.4 \mathrm{~V}$, for example, the 2DEG density is calculated to be $3.0 \times 10^{11} \mathrm{~cm}^{-2}$ or $1.4 \times 10^{11} \mathrm{~cm}^{-2}$ for the Ga-only or the graphene/Ga sample, respectively (Supplementary Fig. 3). The ratio of the two electron densities (2.1) well corresponds to the ratio of channel currents at the same bias (148 nA versus $53 \mathrm{nA}$ ). As bias voltage is increased over the flat band voltage, accumulation electrons build up fast, ensuing electron emission at cathode and space charge build-up in the void channel. In the graphene/Ga sample case, hole space charges are induced in the suspended graphene area and the double injection regime emerges, as evidenced by a steep rise of channel current at $V>$ $0.2 \mathrm{~V}$ (Fig. $3 \mathrm{c}$, blue). Note that the $V^{3}$ regime of the graphene/Ga sample surpasses the $V^{2}$ regime current of the Ga-only sample at $0.8 \mathrm{~V}$. This comparison clarifies the roles played by graphene in different bias regimes: in low bias the work function shift at anode alters the 2DEG density at cathode (therefore, the channel current), whereas in large bias the suspended graphene directly affects cathode emission by inducing hole space charge in the channel, thereby neutralizing electron space charge. 
a

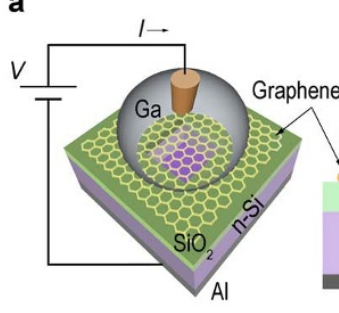

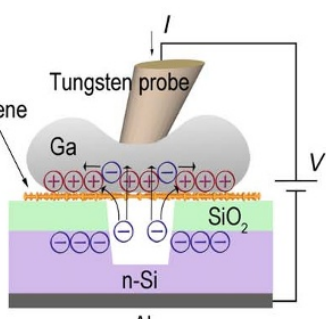

Al

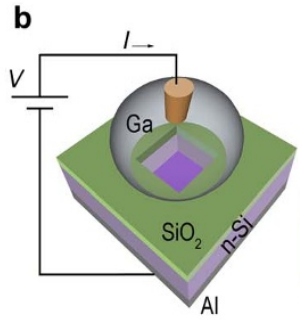

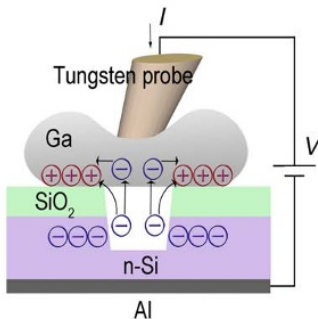

Al

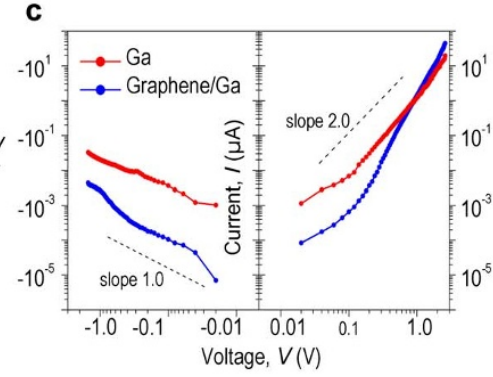

Figure 3 Enhancement of cathode electron emission by a suspended graphene anode. (a), Schematic of a void channel covered with a Ga droplet as a top cover: with a graphene layer placed underneath a Ga cover. (b), Schematic of a void channel covered with a Ga droplet as a top cover: without graphene underneath. (c), Measured $I$ - $V$ characteristics. The void-channel covered with graphene/Ga (blue) shows the Fowler-Nordheim tunneling emission, and the channel current surpasses the Child-Langmuir's space-charge-limited current of the sample without graphene (red) at $V>0.8 \mathrm{~V}$.

Now consider the possible effect of graphene's Fermi level shift on cathode emission, therefore, on the electron capture efficiency at suspended graphene anode. When a Ga droplet is placed on graphene, the graphene's Fermi level is expected to decrease slightly, from $4.56 \mathrm{eV}$ to $4.43 \mathrm{eV}$. At low bias this can make a significant increase of 2DEG density, e.g., at $+0.4 \mathrm{~V}$ from $5.9 \times 10^{10} \mathrm{~cm}^{-2}$ to $1.4 \times 10^{11} \mathrm{~cm}^{-2}$ after placing Ga. At large bias, however, this effect becomes insignificant, e.g., at $+1.0 \mathrm{~V}$ bias the 2DEG density increases from $5.2 \times 10^{11} \mathrm{~cm}^{-2}$ to $6.4 \times 10^{11} \mathrm{~cm}^{-2}$, only 1.2 times increase (Supplementary Fig. 3). The cathode emission of the Gacovered graphene sample is then estimated to have been affected by the same ratio. Overall the result confirms good transparency of monolayer graphene to very low energy electrons that up to $\sim 99.9 \%$ of incident electrons transmit through a suspended graphene electrode. This high level of electron transparency would be beneficial for low leakage current when a suspended graphene is utilized as a control gate (grid) in vacuum electronic devices.

Lastly we elucidate the nature of electron capture at a suspended graphene anode. Here an outstanding question is whether electron capture occurs over the entire area of suspended graphene or only at the channel edges. Previously we reported that in a nano-void-channel MOS with an open-apertured anode the 2DEG emission at cathode is proportional to the perimeter of channel edges, not the area of channel cross-section, and also that injected electrons are captured at the edges of $2 \mathrm{D}$ hole system at anode $\mathrm{e}^{18}$. When a suspended graphene is used as an anode, a new question arises as to whether capture occurs in a fashion highly localized (at the edges) or more uniformly distributed (across the suspended area). In order to answer this question the following samples were prepared and compared. Trench or twin-well structures were fabricated by photolithography and inductively-coupled-plasma reactive ion etching (ICP-RIE). The channel perimeter-to-area ratio was varied in a wide range by employing different channel widths $(5 \mu \mathrm{m}$ or $60 \mu \mathrm{m})$ (Fig. $4 \mathrm{a})$ : Sample 1 with three 5 - $\mu \mathrm{m}$-width trenches $\left(15 \mathrm{~mm}\right.$ perimeter, $3.8 \times 10^{4} \mu \mathrm{m}^{2}$ area) (blue); Sample 2 with one $60-\mu \mathrm{m}$-width trench $(5 \mathrm{~mm}, 1.5 \times$ $10^{5} \mu \mathrm{m}^{2}$ ) (green); and Sample 3 with twin-wells of $50 \mu \mathrm{m} \times$ $25 \mu \mathrm{m}\left(0.3 \mathrm{~mm}, 2.5 \times 10^{3} \mu \mathrm{m}^{2}\right)$ (red). The measured channel currents were normalized by channel perimeter (Fig. $4 \mathrm{~b}$ ).

In all three samples, the channel current reveals a space-charge limited effect ( $V^{1.5}$ regime) in low bias, followed by a steep rise at $V>$ 0.4 or $0.8 \mathrm{~V}$. Here the fast rising regime corresponds to the FowlerNordheim (FN) tunneling emission of electrons at cathode ${ }^{35}$ (Supplementary Fig. 4). Observation of FN emission at large bias implies that the space charge field of electrons at cathode surface is now better (more fully) compensated by hole charges induced in graphene. Note that the degree of space charge neutralization depends on the availability of hole charges in the graphene cover. The near-perfect cancellation of electron space charge field in the three samples can be ascribed to the use of wider channel width ( $5 \mu \mathrm{m}$ or $60 \mu \mathrm{m}$ as opposed to $500 \mathrm{~nm}$ ), which allows more hole charges than before (the 500-nm FIB sample discussed above) for space charge neutralization. The channel current is primarily
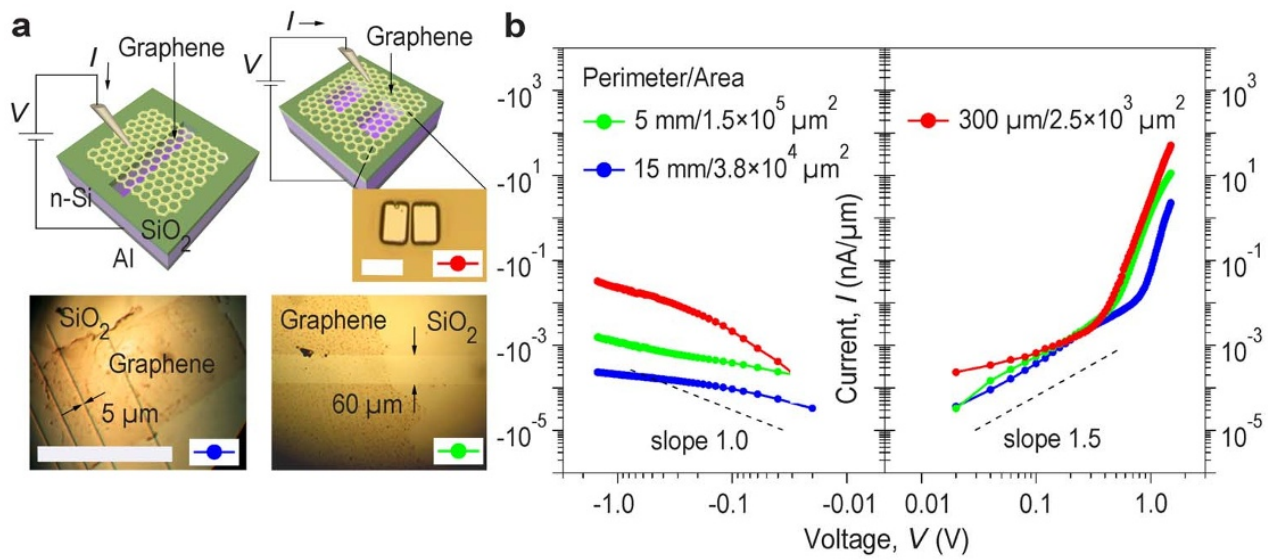

Figure $4 \mid$ Channel-perimeter dependence of electron capture at graphene anode. (a), Schematic of trench-etched void-channels covered with graphene and optical micrographs of 5 - $\mu \mathrm{m}$-width trenches (bottom left) or a $60-\mu \mathrm{m}$-width trench (bottom right), or a twin-well (inset). Scale bars, $3 \mathrm{~mm}$ (bottom) and $50 \mu \mathrm{m}$ (inset). (b), Measured $I-V$ characteristics of trench-etched structures with different perimeter/area ratios. The channel currents normalized by perimeter show a good overlap, indicating that electron capture at graphene anode occurs near/at channel edges, not over the area of graphene. The Fowler-Nordheim regime at larger bias indicates that electron space charge is well neutralized by hole space charge induced in the suspended graphene area. 
determined by two factors, emission of 2DEG at cathode and capture of incident electrons at anode. Cathode emission is perimeterdependent, as reported before ${ }^{18}$. Anode capture would be either perimeter-dependent (if captured at edges) or area-dependent (if captured over the suspended area), with the capture efficiency at graphene edges expected to be much greater than that of free-standing graphene. The perimeter-normalized current of the $V^{1.5}$ regime reveals the same level for the three samples, independent of area (Fig. 4b). This strong perimeter-dependence indicates that in low bias electron capture occurs at graphene edges. At large bias, the FN regime currents of Samples 2 \& 3 (Fig. 4b, green \& red) also show a good overlap, despite a difference in their area/perimeter ratios (i.e., $1.5 \times 10^{5} \mu \mathrm{m}^{2} / 5 \mathrm{~mm}$ versus $2.5 \times 10^{3} \mu \mathrm{m}^{2} / 0.3 \mathrm{~mm}$ ). This confirms strong perimeter-dependence of electron capture at large bias as well. In Sample 1 ( 5 - $\mu \mathrm{m}$ width channels) the $\mathrm{FN}$ regime emerges at higher voltage $(>0.8 \mathrm{~V})$ (Fig. $4 \mathrm{~b}$, blue). This is ascribed to that the relatively small channel width allows a smaller amount of hole space charges compared to the $60-\mu \mathrm{m}$ width samples and therefore requires larger bias to attain the same level of space charge field cancellation. Overall this comparison elucidates that electron capture occurs highly localized to channel edges of graphene.

\section{Discussion}

We characterized electron transparency and hole charge induction response of a suspended graphene electrode on top of a void channel formed in $\mathrm{a} \mathrm{SiO}_{2} / \mathrm{Si}$ substrate. A small fraction $(>\sim 0.1 \%)$ of impinging electrons are captured at the edge of $2 \mathrm{D}$ hole system in graphene, demonstrating good transparency (up to $\sim 99.9 \%$ ) to very low energy $(<3 \mathrm{eV})$ electrons. In response to electron injection, a graphene anode induces hole charges in the suspended area thereby neutralizing electron space charge. This charge compensation dramatically enhances $2 \mathrm{D}$ electron gas emission at cathode to the level far surpassing the Child-Langmuir's space-charge-limited emission. Besides electron transparency, graphene's ability to overcome the space charge limit in cathode emission offers promising potential for low-voltage, high-current-density nanoscale vacuum electronic devices.

\section{Methods}

The graphene/ $\mathrm{SiO}_{2} / \mathrm{Si}$ (GOS) structure with a nano-void channel was fabricated by employing either a focused-ion-beam (FIB) etching technique or a photolithography and plasma reactive-ion etching (RIE) process. First, a $\mathrm{SiO}_{2}$ layer $(\sim 20 \mathrm{~nm}$ thickness) was thermally grown on $n$-type (100)-Si substrate (phosphorous-doped, $5 \Omega$-cm resistivity; $525 \mu \mathrm{m}$ thickness). In the case of FIB etching, square wells were etched into the $\mathrm{SiO}_{2} / \mathrm{Si}$ substrate with a dual beam FIB system (Seiko SMI-3050SE). A Ga-ion beam ( $30 \mathrm{keV} ; 94 \mathrm{pA}$ ) was used with $0.5 \mu$ s dwell time in creating square wells (crosssection, $0.5 \times 0.5 \mu \mathrm{m}^{2}$; etch depth, $\left.1 \mu \mathrm{m}\right)^{18}$. In the case of RIE, a 50-nm-thick Cr layer was deposited on top of the $\mathrm{SiO}_{2}$ layer by thermal evaporation. A window of narrow stripe patterns (5 or $60 \mu \mathrm{m}$ width; single or multiple channels of 8-10 mm length) was then opened in the Cr layer by performing photolithography and RIE. The Cr window etching was performed in $\mathrm{Cl}_{2} / \mathrm{O}_{2}$ ambient with an inductively-coupledplasma reactive-ion etching (ICP-RIE) system (Unaxis 790 ICP-RIE). Subsequently, a trench etching was performed to 500-1000 nm depth by RIE in $\mathrm{CF}_{4} / \mathrm{O}_{2}$ ambient with use of the $\mathrm{Cr}$ window as an etch mask. The remaining photoresist was removed in acetone, followed by rinse in methanol and DI water. The Cr mask was removed in $\mathrm{Cr}$ etchant $\left[\mathrm{NaOH}: \mathrm{K}_{3} \mathrm{Fe}(\mathrm{CN})_{6}: \mathrm{H}_{2} \mathrm{O}=2 \mathrm{~g}: 6 \mathrm{~g}: 22 \mathrm{ml}\right]$. An Al Ohmic contact was prepared on the back side of $\mathrm{Si}$ substrate by thermal evaporation (Al thickness, $\sim 150 \mathrm{~nm}$ ), followed by annealing at $350^{\circ} \mathrm{C}$. Finally, a monolayer graphene (CVD grown on $25-\mu \mathrm{m}$-thick $\mathrm{Cu}$ foil: purchased from ACS Material) was transferred to the trench-etched $\mathrm{SiO}_{2} / \mathrm{Si}$ substrate. The sample was dried at $\sim 70^{\circ} \mathrm{C}$ for 2 hours to remove any moisture trapped in the void-channel. The thus-fabricated samples revealed graphene membranes that are suspended flat on nano-channel-etched substrate (Supplementary Fig. 1).

$I-V$ characteristics of the graphene/ $\mathrm{SiO}_{2} / \mathrm{Si}$ structure were measured in roomtemperature air ambient, with a semiconductor parameter analyzer (HP4145B) in conjunction with use of a probe station. Tungsten probes (tip radius of curvature, $\sim 2 \mu \mathrm{m}$ ) were used in contacting the top (graphene) and bottom (Al) electrodes. The voltage scan was performed with a step size of $0.02 \mathrm{~V}$.

1. Geim, A. K. \& Novoselov, K. S. The rise of graphene. Nature Mater. 6, 183-191 (2007).
2. Castro Neto, A. H. et al. The electronic properties of graphene. Rev. Mod. Phys. 81, 109-162 (2009).

3. Bunch, J. S. et al. Impermeable atomic membranes from graphene sheets. Nano Lett. 8, 2458-2462 (2008).

4. Ando, T., Fowler, A. B. \& Stern, F. Electronic properties of two-dimensional systems. Rev. Mod. Phys. 54, 437-625 (1982).

5. Eisenstein, J. P., Pfeiffer, L. N. \& West, K. W. Negative compressibility of interacting two-dimensional electron and quasiparticle gases. Phys. Rev. Lett. 68 , 674-677 (1992)

6. Ho, L. H. et al. Ground-plane screening of Coulomb interactions in twodimensional systems: how effectively can one two-dimensional system screen interactions in another. Phys. Rev. B 80, 155412 (2009).

7. Li, L. et al. Very large capacitance enhancement in a two-dimensional electron system. Science 332, 825-828 (2011).

8. Kuhr, J.-Ch. \& Fitting, H.-J. Monte Carlo simulation of electron emission from solids. J. Electr. Spectr. Rel. Phenom. 105, 257-273 (1999).

9. Müllerová, I. et al. Very low energy scanning electron microscopy of free-standing ultrathin films. Mater. Transact. 51, 265-270 (2010).

10. Cazaux, J. Reflectivity of very low energy electrons $(<10 \mathrm{eV})$ from solid surfaces: physical and instrumental aspects. J. Appl. Phys. 111, 064903 (2012).

11. Crespi, V. H. et al. Anisotropic electron-beam damage and the collapse of carbon nanotubes. Phys. Rev. B 54, 5927-5931 (1996)

12. Krasheninnikov, A. V. \& Nordlund, K. Ion and electron irradiation-induced effects in nanostructured materials. J. Appl. Phys. 107, 071301 (2010).

13. Child, C. D. Discharge from hot CaO. Phys. Rev. 32, 492-511 (1911).

14. Langmuir, I. The effect of space charge and residual gases on thermionic currents in high vacuum. Phys. Rev. 2, 450-486 (1913)

15. Spindt, C. A. et al. Physical properties of thin-film field emission cathodes with molybdenum cones. J. Appl. Phys. 47, 5248-5263 (1976).

16. Han, J.-W., Oh, J. S. \& Meyyappan, M. Vacuum nanoelectronics: back to the future? - gate insulated nanoscale vacuum channel transistor. Appl. Phys. Lett. 100, 213505 (2012)

17. Stoner, B. R. \& Glass, J. T. Nanoelectronics: nothing is like a vacuum. Nature Nanotechnol. 7, 485-487 (2012).

18. Srisonphan, S., Jung, Y. S. \& Kim, H. K. Metal-oxide-semiconductor field-effect transistor with a vacuum channel. Nature Nanotechnol. 7, 504-508 (2012).

19. Spindt, C. A. A thin-film field-emission cathode. J. Appl. Phys. 39, 3504-3505 (1968).

20. Brodie, I. Physical considerations in vacuum microelectronics devices. IEEE Trans. Electron Devices 36, 2641-2644 (1989).

21. Spindt, C. A. et al. Field-emitter arrays for vacuum microelectronics. IEEE Trans. Electron Devices 38, 2355-2363 (1991).

22. Lampert, M. A. \& Rose. Volume-controlled, two-carrier currents in solids: the injected plasma case. Phys. Rev. 121, 26-37 (1961)

23. Mott, N. F. \& Gurney, R. W. Electronic Processes in Ionic Crystals (Oxford Univ Press, New York, 1940).

24. Grinberg, A. A. et al. Space-charge-limited current in a film. IEEE Trans. Electron Devices 36, 1162-1170 (1989).

25. Langmuir, I. \& Kingdon, K. H. Thermionic effects caused by alkali vapors in vacuum tubes. Science 57, 58-60 (1923).

26. Wilson, V. C. Conversion of heat to electricity by thermionic emission. J. Appl. Phys. 30, 475-481 (1959).

27. Novoselov, K. S. et al. Two-dimensional gas of massless Dirac fermions in graphene. Nature 438, 197-200 (2005).

28. Zhang, Y. et al. Experimental observation of the quantum Hall effect and Berry's phase in graphene. Nature 438, 201-204 (2005).

29. Yu, Y.-J. et al. Tuning the graphene work function by electric field effect. Nano Lett. 9, 3430-3434 (2009).

30. Giovannetti, G. et al. Doping graphene with metal contacts. Phys. Rev. Lett. 101, 026803 (2008).

31. Herring, C. \& Nichols, M. H. Thermionic emission. Rev. Mod. Phys. 21, 185-270 (1949).

32. Das, A. et al. Monitoring dopants by Raman scattering in an electrochemically top-gated graphene transistor. Nature Nanotechnol. 3, 210-215 (2008).

33. Xia, F. et al. The origins and limits of metal-graphene junction resistance. Nature Nanotechnol. 6, 179-183 (2011).

34. Yan, R. et al. Determination of graphene work function and graphene-insulator semiconductor band alignment by internal photoemission spectroscopy. Appl. Phys. Lett. 101, 022105 (2012).

35. Fowler, R. H. \& Nordheim, L. Electron emission in intense electric fields. Proc. Royal Society London 119, 173-181 (1928).

\section{Acknowledgments}

This work was supported by the National Science Foundation (grant no. ECCS-0925532) and the Office of Naval Research (grant no. N00014-1310465)

\section{Author contributions}

S.S. carried out device fabrication \& characterization and model calculation. M.K. performed electron microscopy and device processing \& characterization. H.K.K. designed 
the study, provided theoretical guidance, and supervised the entire project. H.K.K. wrote the manuscript with comments from S.S. and M.K.

\section{Additional information}

Supplementary information accompanies this paper at http://www.nature.com/ scientificreports

Competing financial interests: The authors declare no competing financial interests.
How to cite this article: Srisonphan, S., Kim, M. \& Kim, H.K. Space charge neutralization by electron-transparent suspended graphene. Sci. Rep. 4, 3764; DOI:10.1038/srep03764 (2014).

(c) (i) (2) (2) This work is licensed under a Creative Commons Attribution-

NonCommercial-ShareAlike 3.0 Unported license. To view a copy of this license, visit http://creativecommons.org/licenses/by-nc-sa/3.0 